\title{
RESEARCH OF FLANK WEAR IN TURNING OF STEEL CK60
}

\author{
ZEQIRI, H[akif] M[ehmet]; QEHAJA, N[exhat] E.; ZEQIRI, F[itim]; \\ SALIHU, A[vdi] H[ajdin] \& OSMANI, H[ysni]
}

\begin{abstract}
In this paper are given the researched results of MCT flank wear with strong cutting blades P30, during the turning of Ck60 steel, with change of cutting regimes $(v, s, a)$, without cooling means. It is given the way of work piece and MCT clamping, basic wear parameters, criteria for wear assessment, types, forms and ways of wear and the criteria for its assessment. From obtained results, mathematical models and graphical interpretation, it is given the conclusion for analyzed factors. Processing is performed in turning machine IK62, while the wear was measured with a digit microscope of type JLC680148. As research method was used the statistical method with three factors: $Y=2^{k}+N_{o}$

Keywords: cutting, turning, parameters, tool, flak wear
\end{abstract}

\section{INTRODUCTION}

The knowledge of mechanism of metal- cutting tool wear in the function of cutting regimens, geometry of metal-cutting tool, metal cutting tool and work piece material, time of processing and emulsion has a significant role during the projection of technological process $[1,2,3]$.

The process of flank wear is a result of reaction of different forms of energy such as: mechanical energy, chemical, electric, magnetic and temperature.

The assessment of flank wear obtuseness of metalcutting tool is done through these indicators:

1. Technological indicators, which determine the change of quality manufacturing, mainly the accuracy of dimensions, form and position and as well as parameters of the profile roughness of processed surface, fig. 1.

2. Geometric indicators, which describe stereo metric characteristics of metal-cutting tool, fig. 2.

3. Energy indicators, which pertain to the change of energy relations in decohosation process, which means that the increase of cutting forces requires greater cutting power, which results in the increase of temperature in the cutting process and oscillations of the machine-clamping tool-metal cutting tool- processed particle system.

Type of the processing for ex.: the same material can have a good processing during the clean processing and it can have poor processing during the rough processing, has also a significant role. Consumption of metal-cutting tool is hard process, it is attended by high physicalchemical changes of cutting surface and blade of metalcutting tool which frictions with chip and the surface of processed piece in dry or wet condition. It changes the geometry and form of metal-cutting tool which are closely linked with cutting forces, temperature, plastic deformation and surface roughness.

Consumption process of metal-cutting tool blades can be followed with many following parameters which are divided in: direct and indirect consumption parameters. Direct parameters are: linear parameters one-dimensional, surface two-dimensional and voluminous threedimensional parameters. During the cutting process, special attention should be paid to the way of clamping of work piece as in the character of consumption of cutting blades from the hard metal it effects the of the machine clamping tool-metal cutting tool-processed particle system. The processing was realized with a clamping of work piece with new cutting blade, without emulsion with changing of $\mathrm{v}, \mathrm{s}, \mathrm{a}$ parameters tab. 1. A criterion for consumption measure was taken $1 \mathrm{~km}$ cycle round cutting longitude, by increasing cutting regimes until the first consumption VB value was obtained.

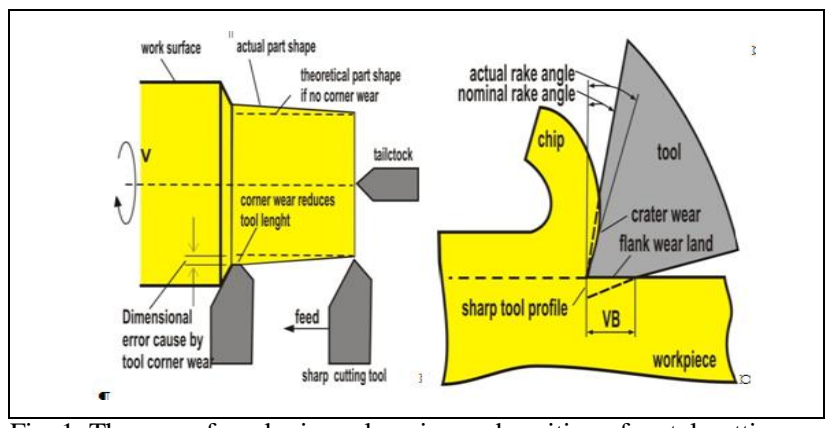

Fig. 1. The way of work piece clamping and position of metal-cutting tool against work piece

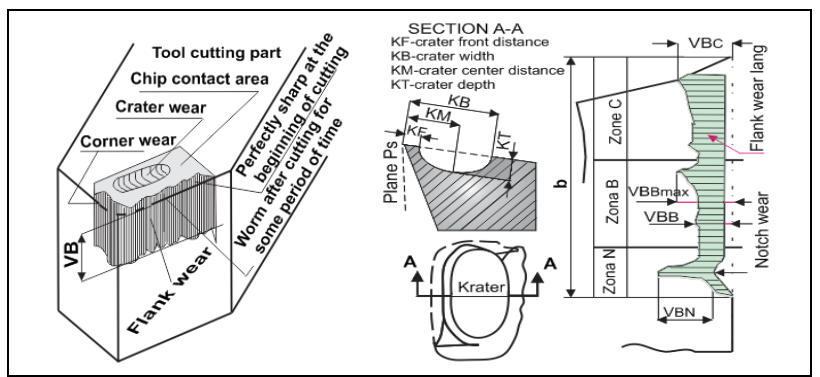

Fig. 2. Geometric indicators of metal-cutting tool consumption

Depending on cutting speed, consumption, is the result of chip friction with flank wear of metal cutting tool. By increasing the cutting speed, high temperatures arise in the cutting zone; by this, the structural changes occur on the surface of metal cutting tool. The crack, particle and plastic deformation can be avoided by selecting appropriate cutting regimes, metal cutting tool material, but this cannot be completely avoided, particularly in metal processing with high durability and hardness, followed by temperature and high mechanical load in cutting area. The wear of metal cutting tool and machine details, is accompanied by mass loss in areas that frictions. By experimental research, it has been proved 
that during the cutting processing at high temperatures, these types of wear are present, fig. 3, 4 .

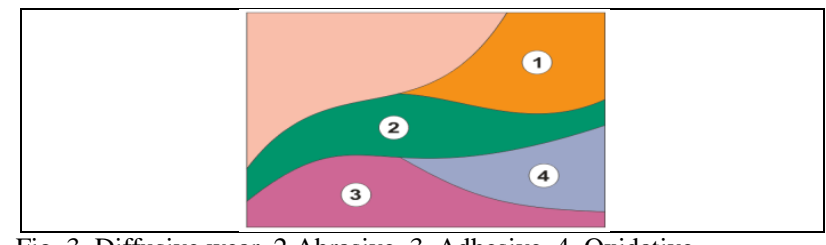

Fig. 3. Diffusive wear, 2.Abrasive, 3. Adhesive, 4. Oxidative

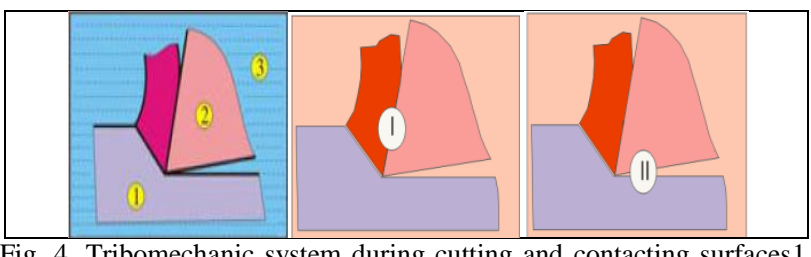

Fig. 4. Tribomechanic system during cutting and contacting surfaces 1 . Cutting piece, 2. metal cutting tool, 3. Means for cooling and lubrication

Thermal wear - occurs due to structural changes by high temperature

Diffusive wear - at high temperature, surfaces of metal cutting tool and chip soften. By this, it comes to the diffuse of these surfaces, which friction, it means, the atoms of metal cutting tool turn into chip. Breaking (fracture) of metal cutting tool, occurs due to high cutting forces (loads) high temperature

Abrasive wear - is a micro-particles layer formed between the surfaces of cutting piece and metal cutting tool. Micro-cuttings are the result of solid particles presence (inter-metalloid, carbides, oxides) in the cutting zone such as: chip particles, flank products, products of burning of alloys elements etc.

Adhesive wear - except abrasive wear, it also comes to fracture of small parts, these fractures are caused to strong alloys, which are sensitive to shock loads. At high temperatures and pressures, as a result of chip adhesion on the front surface of metal cutting tool, small parts detach from this area, which are taken by the chip.

Oxidative wear - at high temperature it comes to the surface oxidation of metal cutting tool, the oxidation layer is very brittle, which is taken away by metal cutting tool in the form of various pieces. Depending on conditions and type of processing with chip remove, friction of front and back surface of metal cutting tool with working piece, the metal cutting tool wear might be:

\section{CRITERIA FOR ASSESSMENT OF METAL CUTTING TOOL WEAR}

Determining the value of rational function of metal cutting tool sustainability is impossible without a reliable criterion for determination of the wear. Criteria for assessing of metal cutting tool's wear are numerous. With the help of these criteria it can be assigned the allowed value of wear, both qualitatively as well as quantitatively. In the theory of chip removal metal processing, there are these criteria for determining the wear of metal cutting tool: Taylor's criterion, Schlesinger's, the rating of wear parameters, optimal wear, radioactive isotopes and technological criteria.

Priority for implementating of one or other criteria is dictated by: production and other circumstances (requirements, needs and purpose). In manufacturing, most often are used: Criterion of wear rating parameters, the criterion of optimal wear and wear technological criteria.

These three criteria are based on experimental researches, which are conducted in direct and systematic order. metal cutting tool is changed when it loses the cutting ability, or when (in the cutting process) the accuracy level of processing (surfaces quality) cannot be ensured. Taylor's Criterion- is the first and oldest criterion for assessing wear flank of metal cutting tool. The determination can be done in experimental manner. So, after a certain cutting time, the back side has to be checked if consumed or not. With wear growth on the back side, it grows: the force of friction of the back side of metal cutting tool with the surface of processed piece. At one point, on the cutting surface of the plastic material it can be observed a bright groove, which is distinguished from the rest of the cut surface. The bright zone on the cut surface is the result of great friction and pressure of metal cutting tool on the processing surface. At the moment when the bright zone is observed, the cutting process must stop because the metal cutting tool is before the phase of catastrophic wear. If the cutting process continues further, the metal cutting tool burns.

Schlesinger's criterion- according to this criterion, the metal cutting tool is considered consumed when the angle $\alpha=0$, in this case we have a significant increase of cutting forces. The metal cutting tool is considered consumed if the cutting tangential force Ft increases 20 $\%$, while the axial cutting forces Fa increases $100 \%$, This criterion requires installation of special equipment for measuring of cutting forces during the cutting process in metal cutting machines.

The criteria of rating wear parameters-is suitable for implementation in manufacturing, where mostly used, it is relatively simple, easily measured previously regulated wear parameters. Longitudinally parameters of metal cutting tool wear are measured according to this criterion, in the first place, wear length in the area of the back side of metal cutting tool (b), the average width of VBB wear zone, the width of the consumed zone at the beginning and the end VBN, VBC and the maximum width of consumed zone VBBmax, fig. 2. Wear rates (border or allowed values) are proved or verified based on the researches mentioned earlier, in a systematic manner to certain working conditions and the type of operation.

The criterion of optimal wear- first base for the criterion of optimum wear was given by $\mathrm{N}$. Zorev. Application of this criterion is done in three fundamental areas:

1. Experimental research, during the research of metal editability through function of metal cutting tool durability

2. Manufacturing lines, mass production and large series.

3. Manufacturing lines, over relative exploitation of expensive metal cutting tool.

The purpose of this criterion is to define an optimal value VB0, one of the wear parameters in the curve, for which was achieved a maximum durability of metal cutting tool, which reaches the minimum costs of processing system under given technological conditions or maximum productivity of the manufacturing process. 
Technological criteria - often used in the production process, according to this criterion the metal cutting tool is considered consumed in the cutting process, when the wear reaches the technological value of the wear size. This value is determined on the basis of technical economic criteria, technical - economic limit values, with the following characteristics: surface roughness, the accuracy of processing, the dynamics of cutting process, processing piece thermal deformations and fractures of metal cutting tool because of stiffness and sensitivity. Under this criterion, the metal cutting tool is considered consumed in the moment (although physically the metal cutting tool could continue cutting process), when the wear parameter VB reaches the technological value, for which the surface roughness, falls below the allowed value (tolerance limit).

\section{GENERAL FORM OF METAL CUTTING TOOL WEAR}

In experimental research has been proven that the wear function of metal cutting tool in the back side is identical to the wear of machinery details, in fig. 5 is shown the wear change curve in three characteristic stages, while in fig. 6 . is shown the modes of cutting blade wear.

Phase (I) - at the beginning of cutting process, the wear of metal cutting tool is quite intensive, curve $0-1$, in phase (II), begins the period of normal wear, wear size raises very slowly, curve $1-2$, in phase (III), it comes to increasing with accelerated intensity or catastrophic wear. If cutting process does not stop in this stage, the metal cutting tool burns. The size of metal cutting tool wear much depends on its geometry. For this reason, geometric parameters and shape of the surface of the metal cutting tool should be chosen such, in order to have minimal wear. The geometry of metal cutting tool for which we have minimal wear, is called optimal geometry.

$$
v_{C} \cdot T^{m}=C
$$

According to Taylor, selected wear parameters can be presented in function of cutting conditions and processing regimes with scalable function of forms:

$$
V_{B}=C \cdot v^{z} \cdot s^{y} \cdot a^{x} \cdot T^{p}
$$

With mathematical model (1) of scalable form, it is obtained the joint dependence in three wear stages, it means, VB size depends on the speed of cutting, feed, cutting depth and durability of metal cutting tool. In this way separately for each different parameter $(v, s, a)$ the dependent variable can be defined by expressions:

$$
V_{B}=C_{v} v^{k v}, \ldots . . V_{B}=C_{S} \cdot s^{k s}, \ldots . . V_{B}=C_{a} \cdot a^{k a}
$$

General constants, which complements the mathematical model is obtained from the condition: general equation is partial, two of the three factors required have constant value. Constants obtained in this way:

$$
C_{1}=C_{v} \cdot s^{ \pm s} \cdot a^{\ddagger k a}, \ldots . C_{2}=C_{s} \cdot v^{ \pm k v} \cdot a^{ \pm k a}, \ldots . . C_{3}=C_{a} \cdot v^{ \pm k v} \cdot s^{ \pm s s}
$$

General constants is obtianed with expression:

$$
C=\frac{1}{3}\left(C_{1}+C_{2}+C_{3}\right),
$$

If cutting is conducted with different cutting speed, feed and depth, Taylor's function is difficult because it requires long time experiment, high costs on materials, metal cutting tool, machine tool and continual number of rotations. Extended Taylor's function, according to Walisch, becomes

$$
T=\frac{C_{v}}{v^{\frac{1}{m}} \cdot s^{\frac{1}{n}} \cdot a^{\frac{1}{k}}},
$$

Usually exponents $1 / \mathrm{m}>1 / \mathrm{n}>1 / \mathrm{k}$, it means, cutting speed has the greatest impact on the sustainability of metal cutting tool, whereas the cutting feed and depth have smaller impact.

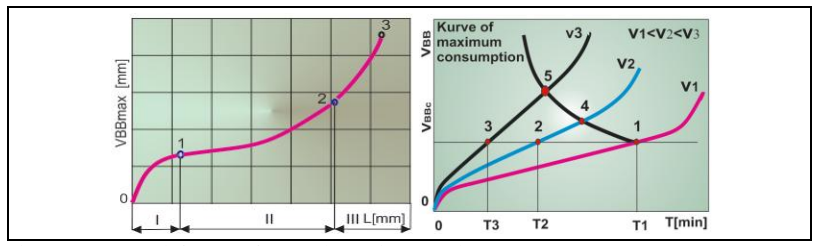

Fig. 5. General form of metal cutting tool wear.

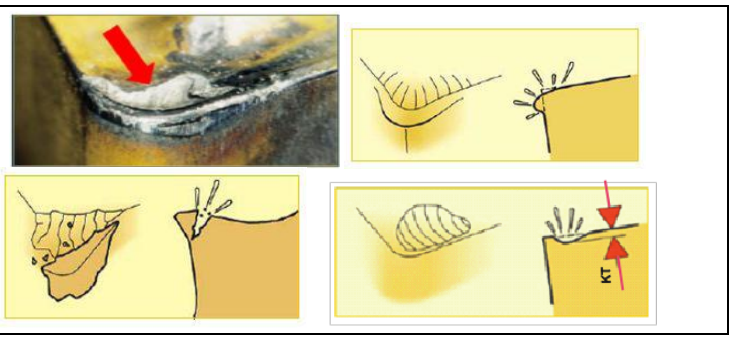

Fig. 6. Modes of cutting blade wear

\section{CONDITIONS DURING THE EXPERIMENT}

1. Tool-Cutting plate from the hard metal P30, SINTALZAGREG, ISO SNMM120404, enforced in the body with sign, ISO PSDNN2525P12, outcome $25 \mathrm{~mm}$, with cutting geometry: $\chi=750, \chi 1=150, \gamma=-60, \alpha=60, \lambda=-60, r \varepsilon=0.4 \mathrm{~mm}$, $\mathrm{bf}=0.2 \mathrm{~mm}, \gamma \mathrm{f}=-200[4]$.

2. Machine-Horizontal Lathe IK62, with these characteristics: $\mathrm{P}=10 \mathrm{~kW}, \mathrm{n}=12.5 \div 2000 \quad \mathrm{rot} / \mathrm{min}, \quad$ and feed $\mathrm{s}=0.035 \div 2.08 \mathrm{~mm} / \mathrm{rev}$.

3. Researching material-Steel Ck60 in the cylinder shape with dimensions $\Phi 68 \times 750 / \Phi 38.5 \times 750 \mathrm{~mm}, \mathrm{Rm}=740 \div 890$ $\mathrm{N} / \mathrm{mm} 2$.

4. Apparatus for roughness measurement-Duplex Microscope CARL SEISS type STOLLBERG/ERCGEB TYP S15A/GA6Og

5. Experimental researching plan - based on the rating number of the rotations of the machine, consulted literature, professional experience, chemical composition, mechanic features of processed piece and metal cutting tool are defined cutting conditions: v, s, a Tab.1.

\begin{tabular}{|c|l|c|c|c|c|}
\hline \multicolumn{7}{|c|}{ Characteristics of indpendent various sizes } \\
\hline \multirow{2}{*}{$\mathrm{Nr}$} & Note & $\begin{array}{c}\text { Level } \\
\text { Code }\end{array}$ & $\begin{array}{c}\text { Maximal } \\
1\end{array}$ & $\begin{array}{c}\text { Average } \\
0\end{array}$ & $\begin{array}{c}\text { Minimal } \\
-1\end{array}$ \\
\hline \multirow{2}{*}{1} & $\mathrm{v}[\mathrm{m} / \mathrm{min}]$ & $\mathrm{X}_{1}$ & 213.00 & 146.000 & 106.000 \\
2 & $\mathrm{~s}[\mathrm{~mm} / \mathrm{rev}]$ & $\mathrm{X}_{2}$ & 0.097 & 0.0685 & 0.0485 \\
3 & $\mathrm{a}[\mathrm{mm}]$ & $\mathrm{X}_{3}$ & 1.500 & 0.866 & 0.500 \\
\hline
\end{tabular}

Tab. 1. Conditions for experiment realization

\begin{tabular}{|l|l|l|}
\hline$Z-1$ & Real plan of matrica & Results \\
\hline
\end{tabular}




\begin{tabular}{|l|c|c|c|c|c|}
\hline & $\begin{array}{c}\mathrm{v} \\
{[\mathrm{m} / \mathrm{min}]}\end{array}$ & $\begin{array}{c}\mathrm{s} \\
{[\mathrm{mm} / \mathrm{rev}]}\end{array}$ & $\begin{array}{c}\mathrm{a} \\
{[\mathrm{mm}]}\end{array}$ & $\begin{array}{c}\mathrm{VB} \\
{[\mu \mathrm{m}]}\end{array}$ & $\operatorname{lnVB}$ \\
\hline 1 & 106 & 0.0485 & 0.500 & 27.876 & 3.327 \\
2 & 213 & 0.0485 & 0.500 & 41.499 & 3.725 \\
3 & 106 & 0.0970 & 0.500 & 30.885 & 3.430 \\
4 & 213 & 0.0970 & 0.500 & 64.318 & 4.163 \\
5 & 106 & 0.0485 & 1.500 & 37.239 & 3.617 \\
6 & 213 & 0.0485 & 1.500 & 42.054 & 3.738 \\
7 & 106 & 0.0970 & 1.500 & 38.746 & 3.657 \\
8 & 213 & 0.0970 & 1.500 & 79.605 & 4.377 \\
9 & 146 & 0.0685 & 0.866 & 37.851 & 3.633 \\
10 & 146 & 0.0685 & 0.866 & 37.851 & 3.633 \\
11 & 146 & 0.0685 & 0.866 & 37.527 & 3.625 \\
12 & 146 & 0.0685 & 0.866 & 37.527 & 3.625 \\
\hline
\end{tabular}

Tab. 2. Derived results during experiment realization

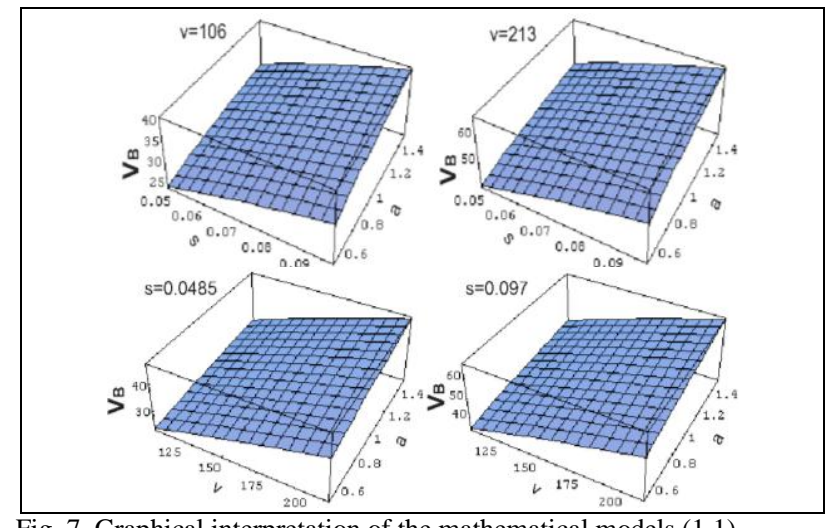

Fig. 7. Graphical interpretation of the mathematical models (1.1)

\section{THE ANALYSIS OF OBTAINED RESULTS}

Upon defining the inlet and outlet parameters, selection of appropriate method for measurement and measuring apparatus, selection of form of function for defining the scope of experiment, statistical processing of researched parameters and verification of mathematical models, the impact of obtained parameters (v, s, a) in consumption magnitude is defined $[4,5,6]$.

During the research was conducted the change of consumption VB in cutting blades from hard metal P30. For describing of this change was adopted the mathematical model of exponential form (1):

$$
V_{B}=C \cdot v^{x} \cdot s^{y} \cdot a^{z}
$$

Consumption of metal-cutting tool is a complex process; it conducts in difficult conditions during cutting it is attended with physical-chemical changes of work piece surface and cutting blade, it changes the geometry of metal-cutting tool which frictions with chip and cutting surface in dry or wet condition. It is closely linked with chip plastic deformation, cutting forces, surface roughness and temperature while cutting.

Mathematical model (6) indicates the impact of cutting regimes in the form and consumption magnitude $\mathrm{VB}$, increasing the cutting speed it changes the conditions in the cutting zone, the chip departs fast from the cutting zone, by this plastic deformations in the chip deformation zone and surface layer zone are reduced, which affects in reducing of non planes. Processing of obtained results during the research as per experimental plan tab. $1 \& 2$, mathematical model (6) is:

$$
V_{B}=4.09059 \cdot v^{0.705} \cdot s^{0.450} \cdot a^{0.169}
$$

From (7) it is clearly seen that in the consumption magnitude VB, the cutting speed (v) and cutting feed (s) have a dominant impact, while the cutting depth (a) has a smaller impact.

Increasing the cutting speed $(\mathrm{v})$ causes reducing of touching surface between the chip and front surface of cutting blade. By this the mechanical and heat impact shifts to the cutting blade by causing higher specific pressures and speed gradient. Friction coefficient between the chip and front surface, chip compression coefficient and cutting forces will be reduced.

Therefore, increasing the cutting speed (v) causes increasing of work from chip friction with front surface of metal-cutting tool. From this it turns out that the cutting speed (v) has a significant impact in consumption magnitude, as the high heat concentration in the cutting blade causes the change not only to mechanical attributes of cutting material but also to cutting tool. Increasing the cutting feed (s) causes increase of removed layer thickness $(\mathrm{s} \sin \chi)$, by this a higher energy is needed to transform it to the chip

\section{CONCLUSION}

Metal-cutting tool consumption is one of the most negative forms in cutting process; it is conducted in difficult conditions depending on cutting regimes and processing technology. Based on analysis of obtained results with experimental research tab. 2, mathematical model (7), and its graphical interpretation fig. 7, we can conclude that:

1. Change of consumption magnitude can be described with function of exponential form;

2. Increasing the cutting speed, consumption of metal-cutting tool will be increase and metal-cutting tool durability will be reduced;

3. Increasing of cutting feed and depth have less impact on consumption magnitude;

4. Presented function might be applied for assigning of boundary fields, choosing of optimal cutting regimes for gaining the desired accuracy and useful economical relations.

\section{REFERENCES}

[1] Bodinaku, A. (2006), Mechanical technology, Volume 2, Faculty of Mechanical Engineering, Tirana, Albania

[2] Bushati, A.: (1979), Mechanical Technology, volume 1, Faculty of Mechanical Engineering, Tirana, Albania

[3] Stankov, J. (1982). Measurement technical basic, methods and experiments planning, FTN, Novi Sad

[4] Milton C. Shaw (2005). Metal cutting principles, Arizona state University, ISBN 0-19-514206-3, Oxford, New York

[5] Salihu, A. (2001), Research of machinability of cutting material with increased speed, doctoral dissertation, Faculty of Mechanical Engineering, Prishtina

[6] Zeqiri, H. (2005), Research of machinability by turning of $42 \mathrm{CrMo} 4$ steel, doctoral dissertation, Faculty of Mechanical Engineering, Prishtina

[7] Salihu, A.; Zeqiri, H.; Bunjaku, A.; Qehaja, N.; Osmani, H. \& Kycyku, A. (2009). Research of the Horizontal Parameters of the Roughness Machined Surface by Turning, Annals of DAAAM for 2009 \& Proceedings of the 20th International DAAAM Symposium, 25-28th November 2009, Vienna, Austria, ISSN 1726-9679, ISBN 978-3-901509-70-4, Katalinic, B. (Ed.), pp. 0119-0120, Published by DAAAM International Vienna, Vienna 